\title{
Stability of Solutions to a Free Boundary Problem for Tumor Growth
}

\author{
Shihe Xu \\ School of Mathematics and Information Sciences, Zhaoqing University, Zhaoqing, Guangdong 526061, China \\ Correspondence should be addressed to Shihe Xu; shihe56789@163.com
}

Received 5 February 2014; Accepted 14 May 2014; Published 21 May 2014

Academic Editor: Gershon Wolansky

Copyright (C) 2014 Shihe $\mathrm{Xu}$. This is an open access article distributed under the Creative Commons Attribution License, which permits unrestricted use, distribution, and reproduction in any medium, provided the original work is properly cited.

We study the asymptotic behaviour of quasi-stationary solutions of a free boundary problem which had been discussed by Bueno (2005). Using a simpler method we prove that the quasi-steady solutions of the problem converge uniformly to the unique nontrivial steady solution.

\section{Introduction}

Tumor progression is a complex process. Understanding its dynamics is one of the great challenges of modern medical science. To describe the growth of solid tumors, an increasing number of mathematical models in forms of free boundary problems of partial differential equations have been proposed and studied during the past several decades (see e.g., [1-6] and the references cited therein). Analysis of such mathematical models has drawn great interest, and many results have been established, compared with [7-17] and references therein. Analysis of such models can help us to examine and distinguish different functions of different mechanisms involved in tumor-growth process and may also assist us in assessing effects of various drug treatments and chemotherapy.

In this work we give a remark to the asymptotic behaviour of quasi-stationary solutions of a free boundary value problem that models the growth of a single nonnecrotic tumour, which is supposed to be spherical. The model was initially proposed by Byrne and Chaplain [3] and recently was studied by Bueno et al. [7]. In [7] the authors established the existence of a nontrivial steady solution and conditions for the existence and uniqueness of a quasi-steady solution for each initial configuration. The authors also proved that all these quasi-steady solutions converge uniformly to a nontrivial steady solution. In this paper, we use a different method to prove that all these quasi-steady solutions converge uniformly to a nontrivial steady solution.

The general model is as follows:

$$
\begin{gathered}
c \frac{\partial \sigma}{\partial t}=\frac{1}{r^{2}} \frac{\partial}{\partial r}\left(r^{2} \frac{\partial \sigma}{\partial r}\right)-f(\sigma(r, t)), \quad 0<r<R(t), t>0, \\
\frac{\partial \sigma}{\partial r}(0, t)=0, \quad \sigma(R(t), t)=\bar{\sigma}, \quad t>0, \\
R^{2} R^{\prime}(t)=\int_{0}^{R(t)} S(\sigma(r, t)) r^{2} d r, \quad t>0, \\
\sigma(r, 0)=\sigma_{0}(r), \quad 0<r<R(0), \\
R(0)=R_{0} .
\end{gathered}
$$

Equation (2) is a (dimensionless) reaction-diffusion equation in $\sigma$, where $\sigma$ is the nutrient concentration and $f(\sigma)$ is the absorption rate. Moreover, with $\bar{\sigma}$ we denoted the external concentration of nutrients. We assume that $f(\sigma) \geq 0$ and $f(\sigma)=0$ only occurs for a single value denoted by $\underline{\sigma}$. Let $\varepsilon:=T_{1} / T_{2}$ be the ratio between the time necessary for the diffusion of nutrients, $T_{1}$, and the time interval, $T_{2}$, elapsed until the tumour doubles its size. Typical values for $T_{1}$ and $T_{2}$ are, respectively, of the order of minutes and days. Thus, 
$\varepsilon \ll 1$. Note that the condition $(\partial \sigma / \partial r)(0, t)=0$ follows from the symmetry of the problem; it is necessary to produce smooth solutions at the origin. $S(\sigma)$ is the proliferation rate function-the balance between the mitosis and death rates generated by deficiency of nutrients (hypoxic apoptosis and necrosis) of the cells. For details, please see [7]. In [7], the authors only considered the case $\varepsilon=0$, and the case $0<$ $\varepsilon \ll 1$ was studied by Cui and Friedman [9]. As that in [7], to simplify the notation, we assume that $\underline{\sigma}=0, \bar{\sigma}=1$. Then actually the model studied in [7] is as follows:

$$
\begin{gathered}
\frac{1}{r^{2}} \frac{\partial}{\partial r}\left(r^{2} \frac{\partial \sigma}{\partial r}\right)=f(\sigma(r, t)), \quad 0<r<R(t), t>0, \\
\frac{\partial \sigma}{\partial r}(0, t)=0, \quad \sigma(R(t), t)=1, \quad t>0, \\
R^{2} R^{\prime}(t)=\int_{0}^{R(t)} S(\sigma(r, t)) r^{2} d r, \quad t>0, \\
\sigma(r, 0)=\sigma_{0}(r), \quad 0<r<R(0), \\
R(0)=R_{0} .
\end{gathered}
$$

Under the following assumptions

(H1) $f \in C^{1}([0,1] ; R) ; f^{\prime}(\sigma) \geq 0$ for $0 \leq \sigma \leq 1 ; f(\sigma)=$ $0 \Leftrightarrow \sigma=0$

(H2) $S \in C^{1}([0,1] ; R) ; S^{\prime}(\sigma)>0$ for $0 \leq \sigma \leq 1 ; S(\widetilde{\sigma})=$ $0,0<\widetilde{\sigma}<1$

(H3) $S(\widetilde{\sigma})=0$ for some value $\widetilde{\sigma} \in(0,1)$;

the authors established the existence of a nontrivial steady solution and conditions for the existence and uniqueness of a quasi-steady solution for each initial configuration. Also, the authors proved that all these quasi-steady solutions converge uniformly to a nontrivial steady solution. In this paper we use a different method to prove all these quasi-steady solutions converge uniformly to a nontrivial steady solution. The method used in this paper is simpler than that used in [7].

\section{Asymptotic Behavior of the Solutions to (2)-(6)}

From $[7,9]$, we know that if the assumptions (H1), (H2), and (H3) hold, then the system (2)-(4) has a unique stationary solution $\left(\sigma_{s}(r), R s\right)=\left(u\left(r / R_{s}, R_{s}^{2}\right), R_{s}\right)$.

Our main result is the following Theorem.

Theorem 1. Assume that the assumptions (H1), (H2), and (H3) are satisfied and let $(\sigma(r, t), R(t))$ be the solution of the problem (2)-(6). Then for any $R_{0}>0$ one has

$$
\lim _{t \rightarrow \infty} R(t)=R_{s}, \quad \lim _{t \rightarrow \infty} \sigma(r, t)=\sigma_{s}(r) .
$$

First we consider the following boundary value problem:

$$
\begin{gathered}
\Delta_{\rho} u=\lambda f(u), \quad 0<\rho<1, \\
\frac{\partial u}{\partial \rho}(0, \lambda)=0, \quad u(1, \lambda)=1,
\end{gathered}
$$

where $\lambda$ is nonnegative parameter and $\Delta_{\rho} u=$ $\left(1 / \rho^{2}\right)(\partial / \partial \rho)\left(\rho^{2}(\partial u / \partial \rho)\right)$. Existence of a solution $u$ of (8) follows readily from the upper and lower solution method (see [18]) because it is clear that $u=1$ and $u=0$ are a pair of upper and lower solutions. Uniqueness of the solution follows from the fact that the function $f$ is monotone increasing. Denote the solution of $(8)$ by $u(\rho, \lambda)$. Since (2) is autonomous, setting $\rho=r / R$, one can easily check that $u\left(r / R, R^{2}\right)$ is a unique solution of (2)-(3) by the fact that $\sigma(r, t)=u\left(r / R, R^{2}\right)$.

Lemma 2 (see [9, Lemma 3.1]). Assume that the assumption (H1) is satisfied. Then the following assertions hold.

(i) For any $\lambda \geq 0$, the problem (8) has the unique positive solution $u(\rho, \lambda)$, and

$$
\begin{gathered}
0<u(\rho, \lambda) \leq 1 \quad \text { for } 0 \leq \rho \leq 1, \lambda \geq 0, \\
0<\frac{\partial u}{\partial \rho} \leq \frac{1}{3} \lambda \rho f(1) \quad \text { for } 0<\rho \leq 1, \lambda \geq 0 .
\end{gathered}
$$

(ii) $u(\rho, \lambda)$ is continuously differentiable in $\lambda$ for all $0 \leq \rho \leq$ $1, \lambda \geq 0$, and

$$
-\frac{f(1)}{6} \leq \frac{\partial u}{\partial \lambda}<0 \quad \text { for } 0 \leq \rho \leq 1, \lambda \geq 0 .
$$

Lemma 3. Consider the following problem

$$
\begin{gathered}
x^{\prime}(t)=x(t) f(x(t)), \quad t>0, \\
x(0)=x_{0}>0 .
\end{gathered}
$$

Assume that $f$ is defined and continuously differentiable on $(0, \infty)$ and $f^{\prime}(x)<0$ for all $x>0$. If there exists a unique positive constant $x_{s}$ such that $f\left(x_{s}\right)=0$, then there holds

$$
\lim _{t \rightarrow \infty} x(t)=x_{s} \text {. }
$$

Proof. It is obvious there exists a unique solution to the problem (11). In the following we prove the asymptotic behavior of this solution. First we claim that if $x_{0}<x_{s}$, then $x(t)<x_{s}$ for all $t>0$. If not, there exists a point $t_{0}$ such that

$$
x\left(t_{0}\right)=x_{s}, \quad x^{\prime}\left(t_{0}\right)>0, \quad x(t)<x_{s} \quad \text { for } t<t_{0} .
$$

On the other hand, noticing the conditions (H2) and (H3), we obtain

$$
x^{\prime}\left(t_{0}\right)=x\left(t_{0}\right) f\left(x\left(t_{0}\right)\right)=x_{s} f\left(x_{s}\right)=0,
$$

which is in contradiction with (13). Thus the claim is true. Then $x(t)$ is monotone increasing and has a upper bound, so $x(t)$ has a limit as $t \rightarrow \infty$. This limit must be equal to $x_{s}$.

If $x_{0}>x_{s}$, the proof is similar.

Proof of Theorem 1. Substituting $\sigma(r, t)=u\left(r / R, R^{2}\right)$ in (4), one can get

$$
\begin{aligned}
R^{\prime}(t) & =\frac{1}{R^{2}} \int_{0}^{R} S(\sigma(r, t)) r^{2} d r=R \int_{0}^{1} S(u(\rho, \lambda)) \rho^{2} d \rho \\
& =: R F(R),
\end{aligned}
$$


where $\rho=r / R, \lambda=R^{2}$. Direct computation yields

$$
\begin{aligned}
F^{\prime}(R)= & \int_{0}^{1} S^{\prime}(u) \frac{\partial u}{\partial \rho}\left(-\frac{r}{R^{2}}\right) \rho^{2} d \rho+2 R \int_{0}^{1} S^{\prime}(u) \frac{\partial u}{\partial \lambda} \rho^{2} d \rho \\
& +\int_{0}^{1} 2 \rho S(u)\left(-\frac{r}{R^{2}}\right) d \rho<0,
\end{aligned}
$$

where we have used the facts that $\partial u / \partial \rho>0$ and $\partial u / \partial \lambda<0$ (see Lemma 2) and $S^{\prime}(u)>0$. By the fact that $F(x)=0$ has a unique positive constant solution $R_{s}$ under the assumptions (H1), (H2), and (H3), by Lemma 3 one can get

$$
\lim _{t \rightarrow \infty} R(t)=R_{s}
$$

for any initial value $R_{0}>0$. By the fact that $\sigma(r, t)=$ $u\left(r / R, R^{2}\right)$ and using Lemma 2 (ii), we have

$$
\begin{aligned}
\mid \sigma & (r, t)-\sigma_{s}(r) \mid \\
& =\left|u\left(\frac{r}{R}, R^{2}\right)-u\left(\frac{r}{R_{s}}, R_{s}^{2}\right)\right| \\
& =\left|u_{\rho}(\xi, \lambda)\left(\frac{r}{R}-\frac{r}{R_{s}}\right)+u_{\lambda}(\rho, \eta)\left(R^{2}-R_{s}^{2}\right)\right| \\
& \leq \frac{f(1)}{3 R_{s}} R r^{2}\left|R(t)-R_{s}\right|+\frac{f(1)}{6}\left|R(t)+R_{s}\right|\left|R(t)-R_{s}\right| \\
& \leq\left|\frac{f(1)}{3 R_{s}} R^{3}+\frac{f(1)}{6}\right| R(t)+R_{s}||\left|R(t)-R_{s}\right|,
\end{aligned}
$$

where $\xi=\theta_{1}(r / R)+\left(1-\theta_{1}\right)\left(r / R_{s}\right), \eta=\theta_{2} R^{2}+\left(1-\theta_{2}\right) R_{s}^{2}$, $\theta_{1}, \theta_{2} \in(0,1)$. Thus $\lim _{t \rightarrow \infty} \sigma(r, t)=\sigma_{s}(r)$ uniformly in $[0, R(t)]$. This completes the proof of Theorem 1 .

In our work, like in [7], due to normalization, we have considered $\underline{\sigma}=0$ and $\bar{\sigma}=1$. We recall that $\underline{\sigma}$ denotes the only zero of the absorption rate $f(\sigma), \bar{\sigma}$ is the concentration of nutrients at the border of the tumour, and $\widetilde{\sigma}$ is the zero of the proliferation rate $S(\sigma)$. We now state our conclusions for regimes of growth in terms of generical values of $\underline{\sigma}$ and $\bar{\sigma}$ and prove them.

We summarize the results as follows (see also in [7]).

(a) If $\underline{\sigma}<\tilde{\sigma}<\bar{\sigma}$, then the nontrivial stationary solution is globally asymptotically stable (and the trivial solution unstable); that is, $R(t) \rightarrow R_{s}$ for any value of $R_{0}=$ $R(0)>0$. This case corresponds to $S(0)<0<S(1)$ and was proven in Theorem 1 .

(b) If $\underline{\sigma}<\bar{\sigma}<\tilde{\sigma}$, then the trivial solution (which is stationary) is globally asymptotically stable; that is, $R(t) \rightarrow 0$ for any value of $R_{0}=R(0)>0$. This case corresponds to $S(0)<S(1)<0$.

(c) If $\widetilde{\sigma}<\underline{\sigma}<\bar{\sigma}$, then $R(t) \rightarrow \infty$ for any value of $R_{0}=$ $R(0)>0$. This case corresponds to $0<S(0)<S(1)$.
Proof of (b). By (4), (15), and Lemma 2 (i), we have

$$
\begin{aligned}
\frac{S(0)}{3} R(t) & =R \int_{0}^{1} S(0) \rho^{2} d \rho \leq R^{\prime}(t) \\
& \leq R \int_{0}^{1} S(1) \rho^{2} d \rho=\frac{S(1)}{3} R(t) .
\end{aligned}
$$

By $(\mathrm{H} 2)$ and $\underline{\sigma}<\bar{\sigma}<\widetilde{\sigma}$, we have $S(0)<S(1)<S(\widetilde{\sigma})=0$; then from (19) we have

$$
\begin{aligned}
R_{0} \exp \left(\frac{S(0) t}{3}\right) \leq R(t) \leq R_{0} \exp \left(\frac{S(1) t}{3}\right) & \longrightarrow 0, \\
t & \longrightarrow \infty .
\end{aligned}
$$

This verifies $R(t) \rightarrow 0$ for any value of $R_{0}=R(0)>0$ as $t \rightarrow \infty$.

Proof of (c). By (4), (15), and Lemma 2 (i), we have

$$
\begin{aligned}
\frac{S(0)}{3} R(t) & =R \int_{0}^{1} S(0) \rho^{2} d \rho \leq R^{\prime}(t) \\
& \leq R \int_{0}^{1} S(1) \rho^{2} d \rho=\frac{S(1)}{3} R(t) .
\end{aligned}
$$

By $(\mathrm{H} 2)$ and $\widetilde{\sigma} \leq \sigma<\bar{\sigma}$, we have $0=S(\widetilde{\sigma})<S(0)<S(1)$; then from (19) we have

$$
R_{0} \exp \left(\frac{S(0) t}{3}\right)<R(t) \leq R_{0} \exp \left(\frac{S(1) t}{3}\right) .
$$

This verifies $R(t) \rightarrow \infty$ for any value of $R_{0}=R(0)>0$ as $t \rightarrow \infty$ since $S(0)>0$.

\section{Conflict of Interests}

The author declares that there is no conflict of interests regarding the publication of this paper.

\section{Acknowledgments}

This work is supported by NSF of China (11226182, 11301474, and 11171295) and Foundation for Distinguished Young Teacher in Higher Education of Guangdong, China (Yq2013163).

\section{References}

[1] M. Bodnar and U. Foryś, "Time delay in necrotic core formation," Mathematical Biosciences and Engineering, vol. 2, no. 3, pp. 461-472, 2005.

[2] F. S. Borgesa, K. C. Iarosza, H. P. Renb et al., "Model for tumour growth with treatment by continuous and pulsed chemotherapy," Biosystems, vol. 116, pp. 43-48, 2014.

[3] H. Byrne and M. Chaplain, "Growth of nonnecrotic tumors in the presence and absence of inhibitors," Mathematical Biosciences, vol. 130, pp. 151-181, 1995.

[4] S. Fu and S. Cui, "Global existence and stability of solution of a reaction-diffusion model for cancer invasion," Nonlinear Analysis. Real World Applications, vol. 10, no. 3, pp. 1362-1369, 2009. 
[5] J. Ward and J. King, "Mathematical modelling of avasculartumor growth-II: modelling grwoth saturation," IMA Journal of Mathematics Applied in Medicine and Biology, vol. 15, pp. 142, 1998.

[6] J. Wu and F. Zhou, "Asymptotic behavior of solutions of a free boundary problem modeling the growth of tumors with fluidlike tissue under the action of inhibitors," Transactions of the American Mathematical Society, vol. 365, no. 8, pp. 4181-4207, 2013.

[7] H. Bueno, G. Ercole, and A. Zumpano, "Asymptotic behaviour of quasi-stationary solutions of a nonlinear problem modelling the growth of tumours," Nonlinearity, vol. 18, no. 4, pp. 1629$1642,2005$.

[8] S. Cui, "Analysis of a mathematical model for the growth of tumors under the action of external inhibitors," Journal of Mathematical Biology, vol. 44, no. 5, pp. 395-426, 2002.

[9] S. Cui and A. Friedman, "Analysis of a mathematical model of the growth of necrotic tumors," Journal of Mathematical Analysis and Applications, vol. 255, no. 2, pp. 636-677, 2001.

[10] S. Cui and S. Xu, "Analysis of mathematical models for the growth of tumors with time delays in cell proliferation," Journal of Mathematical Analysis and Applications, vol. 336, no. 1, pp. 523-541, 2007.

[11] J. Escher and A.-V. Matioc, "Well-posedness and stability analysis for a moving boundary problem modelling the growth of nonnecrotic tumors," Discrete and Continuous Dynamical Systems B, vol. 15, no. 3, pp. 573-596, 2011.

[12] J. Escher and A.-V. Matioc, "Bifurcation analysis for a free boundary problem modeling tumor growth," Archiv der Mathematik, vol. 97, no. 1, pp. 79-90, 2011.

[13] J. Escher and A.-V. Matioc, "Analysis of a two-phase model describing the growth of solid tumors," European Journal of Applied Mathematics, vol. 24, no. 1, pp. 25-48, 2013.

[14] U. Forys and M. Bodnar, "Time delays in proliferation process for solid avascular tumour," Mathematical and Computer Modelling, vol. 37, pp. 1201-1209, 2003.

[15] A. Friedman and F. Reitich, "Analysis of a mathematical model for the growth of tumors," Journal of Mathematical Biology, vol. 38, no. 3, pp. 262-284, 1999.

[16] S. Xu, "Analysis of tumor growth under direct effect of inhibitors with time delays in proliferation," Nonlinear Analysis. Real World Applications, vol. 11, no. 1, pp. 401-406, 2010.

[17] S. Xu and Z. Feng, "Analysis of a mathematical model for tumor growth under indirect effect of inhibitors with time delay in proliferation," Journal of Mathematical Analysis and Applications, vol. 374, no. 1, pp. 178-186, 2011.

[18] J. Smoller, Shock Waves and Reaction-Diffusion Equations, vol. 258, Springer-, New York, NY, USA, 2nd edition, 1994. 


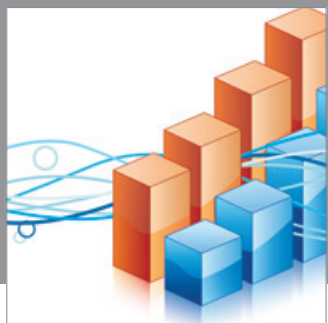

Advances in

Operations Research

mansans

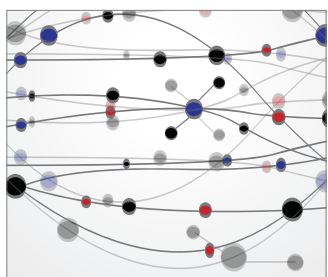

The Scientific World Journal
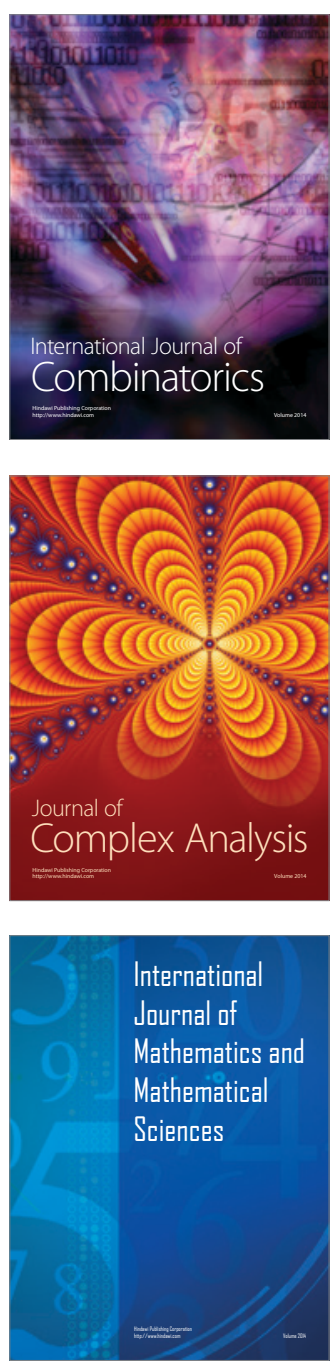
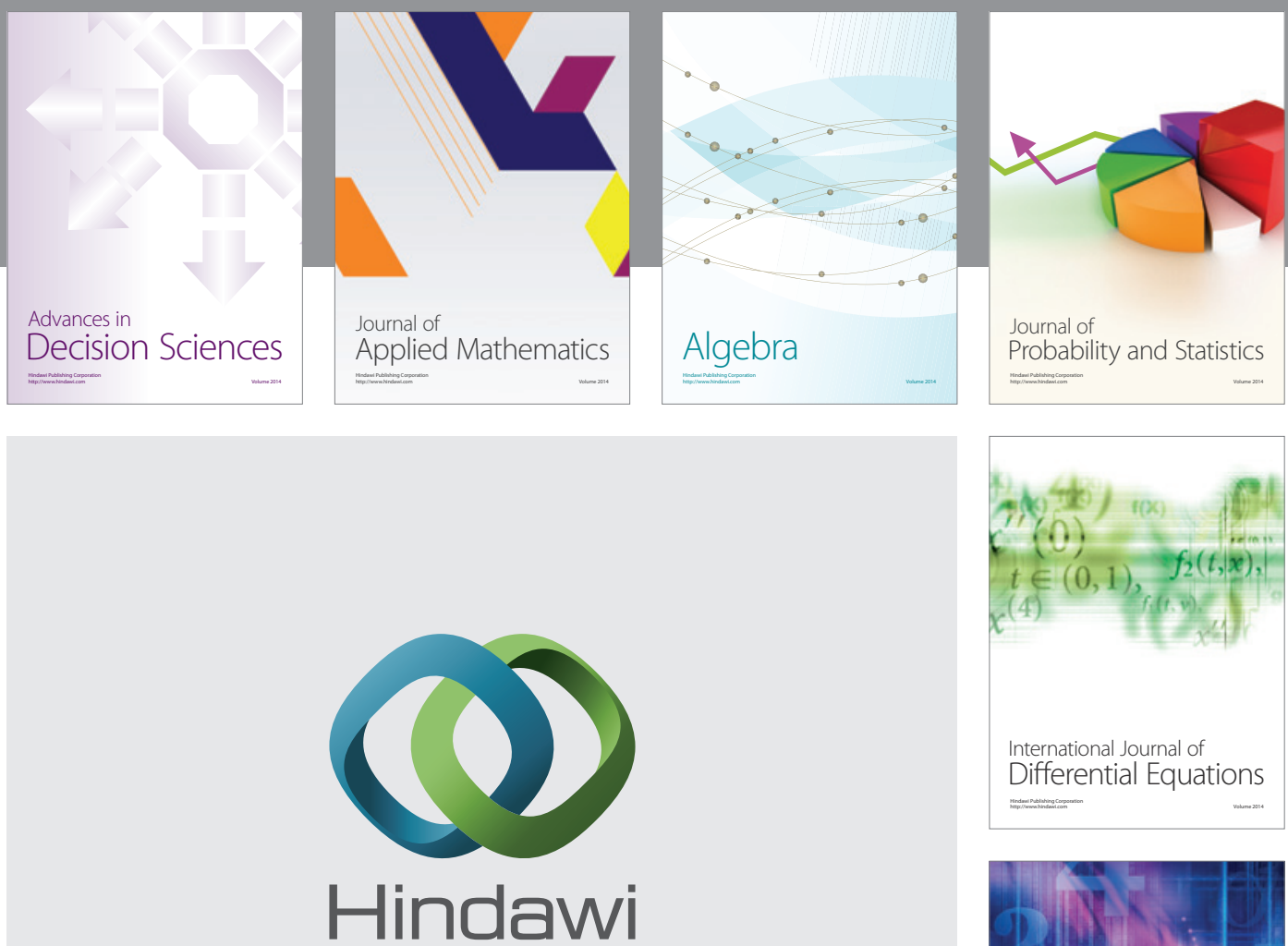

Submit your manuscripts at http://www.hindawi.com
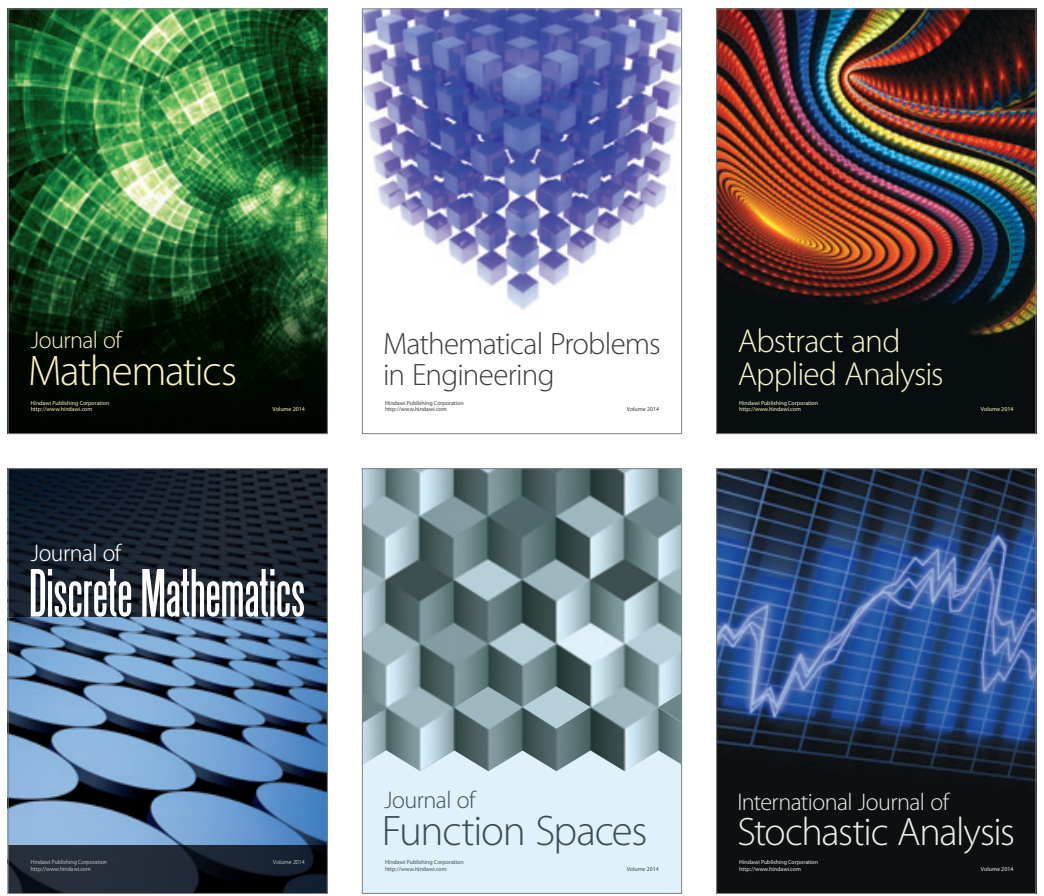

Journal of

Function Spaces

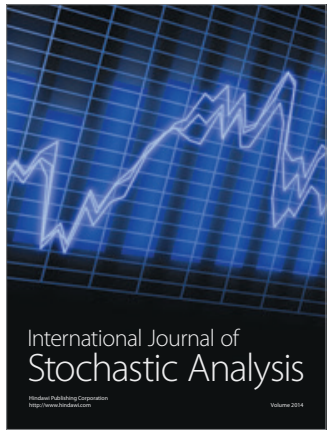

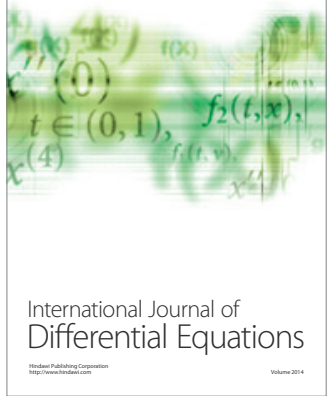
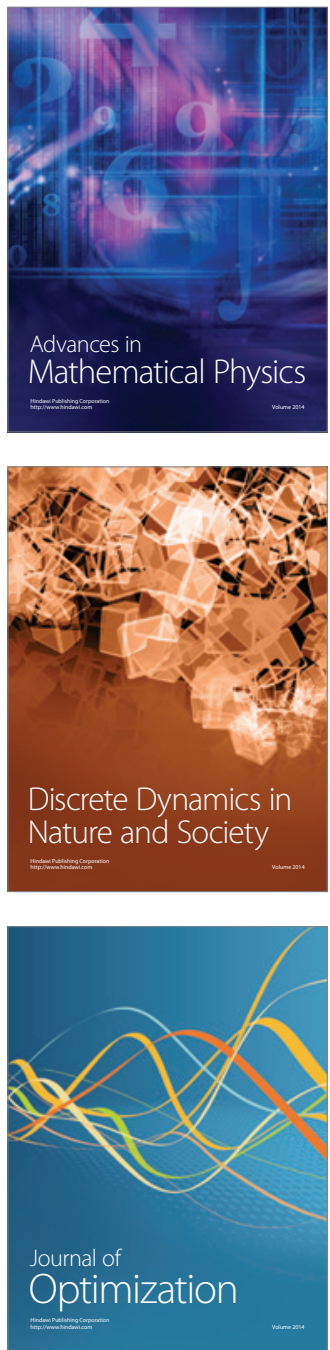